\title{
EDITORIAL
}

\section{A cerebrospinal fluid biosignature for the diagnosis of Alzheimer's disease}

\author{
Tatiana Barichello, ${ }^{1,2}$ iD Vijayasree V. Giridharan, ${ }^{1}$ Felipe Dal-Pizzol ${ }^{2}$ \\ ${ }^{1}$ Translational Psychiatry Program, Department of Psychiatry and Behavioral Sciences, McGovern Medical School, The University of Texas \\ Health Science Center at Houston, Houston, TX, USA. ${ }^{2}$ Laboratório de Fisiopatologia Experimental, Programa de Pós-Graduação em Ciências \\ da Saúde, Universidade do Extremo Sul Catarinense (UNESC), Criciúma, SC, Brazil.
}

\begin{abstract}
Alzheimer's disease (AD) and other dementia (OD) are major and increasing global health challenges. The World Health Organization (WHO) estimates that around 50 million people worldwide have dementia, of which $A D$ is the most common form, potentially contributing to 60 to $70 \%$ of these cases. The alarming global burden of dementia is projected to reach 82 million by 2030 and 152 million people by $2050 .^{1}$
\end{abstract}

$A D$ biomarkers play an essential role in detecting the disease in its early stages, thus allowing initiation of treatment. Being in direct contact with the brain and spinal cord, cerebrospinal fluid (CSF) has been known to act as a valuable source of biomarkers that are representative of various biochemical and metabolic profiles of the brain. The established CSF biomarkers for diagnosis of $A D$ are the 42 -amino acid form of amyloid-beta $\left(A \beta_{42}\right)$ and the total and phosphorylated fractions of tau protein ( $T$-tau and P-tau, respectively). CSF $A \beta_{42}$ levels are low in patients with $A D$ when compared with healthy controls, due to increased amyloid deposition in the brain of these patients. Tau protein is a microtubule-associated protein expressed in neurons, and is considered as an important biomarker of several disorders in which axonal damage is implicated. In $A D$, T-tau and P-tau concentrations in the CSF are elevated due to cortical neuronal loss and cortical tangle formation, respectively. The most recent well-established biomarker for $A D$ diagnosis is the neurofilament light chain (NFL) protein. NFL is a marker of neuroaxonal damage, and its levels are elevated in the CSF of patients with $A D$ and other neurological conditions as compared with healthy controls. These high NFL levels in the CSF are due to prominent axonal destruction triggered by neurodegeneration. A recent systematic review and meta-analysis by Olsson et al. recommends using CSF $A \beta_{42}$, T-tau, P-tau, and NFL levels as a panel of diagnostic biomarkers for AD in clinical practice and research ${ }^{2}$ (Figure 1).

In this issue of the Brazilian Journal of Psychiatry, Radanovic et al. evaluated $A \beta_{1-42}, P$-tau, and T-tau in the CSF of healthy controls $(n=54)$, patients with mild cognitive impairment $(\mathrm{MCl})(\mathrm{n}=82)$, patients with $A D$ $(n=46)$, and patients with OD $(n=26)$, and tested for correlation with Mini-Mental State Examination (MMSE) scores. CSF from patients with $A D$ contained higher levels of P-tau and T-tau and lower levels of $A \beta_{1-42}$, as well as a lower $A \beta_{1-42} / P$-tau ratio, as compared to healthy controls and those with $\mathrm{MCl}$, but not to the $\mathrm{OD}$ group.
By evaluating the coefficient of correlation between CSF biomarkers and MMSE, the authors found that the $A \beta_{1-42}$ levels in the CSF and MMSE scores correlated weakly in the $\mathrm{MCl}(0.247)$, moderately in the OD (0.440), and weakly and inversely $(-0.145)$ in the AD group. T-tau CSF levels had a weak, inverse correlation with MMSE scores in the control $(-0.284)$ and $\mathrm{MCl}(-0.241)$ groups; this correlation was moderate to high in the OD $(-0.665)$ group. $\mathrm{P}$ tau CSF levels presented a weak negative correlation with the AD group (-0.343) and moderate negative correlation with the OD group $(-0.540){ }^{3}$ The authors did not find a strong correlation between CSF biomarkers and MMSE scores; however, an important contribution of this study was to reaffirm the importance of the CSF biomarkers $A \beta_{1-42}, P$-tau, and T-tau to distinguish patients with $A D$ from controls and OD in the Brazilian population.

The limitations of adopting MMSE scores in AD clinical trials was demonstrated by Chapman et al., who examined the diagnostic accuracy of MMSE and Logical Memory (LM) cutoffs used in AD trials and diagnostic studies, tested using subjects from the NACC database diagnosed with normal cognition $(n=10,741), \mathrm{MCl}(n=5,883)$, or $A D$ dementia $(n=6,814)$. The results from this large-cohort study revealed that MMSE and LM scores might not be appropriate tools for the selection of subjects to enroll in multicenter studies designed to develop therapeutics and diagnostic methods for AD. ${ }^{4}$

Another study, by Olsson et al., demonstrated the association between cognition and CSF-NFL levels. Their wide-ranging sample included healthy controls $(n=75)$ and patients with $\mathrm{MCl}(\mathrm{n}=114), A D(n=397)$, frontotemporal dementia (FTD) $(n=96)$, amyotrophic lateral sclerosis $(n=68)$, Parkinson's disease (PD) $(n=41)$, PD with $\mathrm{MCl}$ $(n=19)$, PD dementia $(n=29)$, dementia with Lewy bodies $(n=33)$, corticobasal syndrome $(n=21)$, and progressive supranuclear palsy $(n=20)$. The patients were followed longitudinally for cognitive testing over a period of 1 to 18 years. The CSF biomarkers evaluated included $A \beta_{1-42}$, P-tau, T-tau, and NFL. Compared to other markers, CSF levels of NFL gradually increased among the control, $\mathrm{MCl}$, and $A D$ groups. These results delineate the increased

How to cite this article: Barichello T, Giridharan VV, Dal-Pizzol F. A cerebrospinal fluid biosignature for the diagnosis of Alzheimer's disease. Braz J Psychiatry. 2019;41:467-468. http://dx.doi.org/ 10.1590/1516-4446-2019-0629 


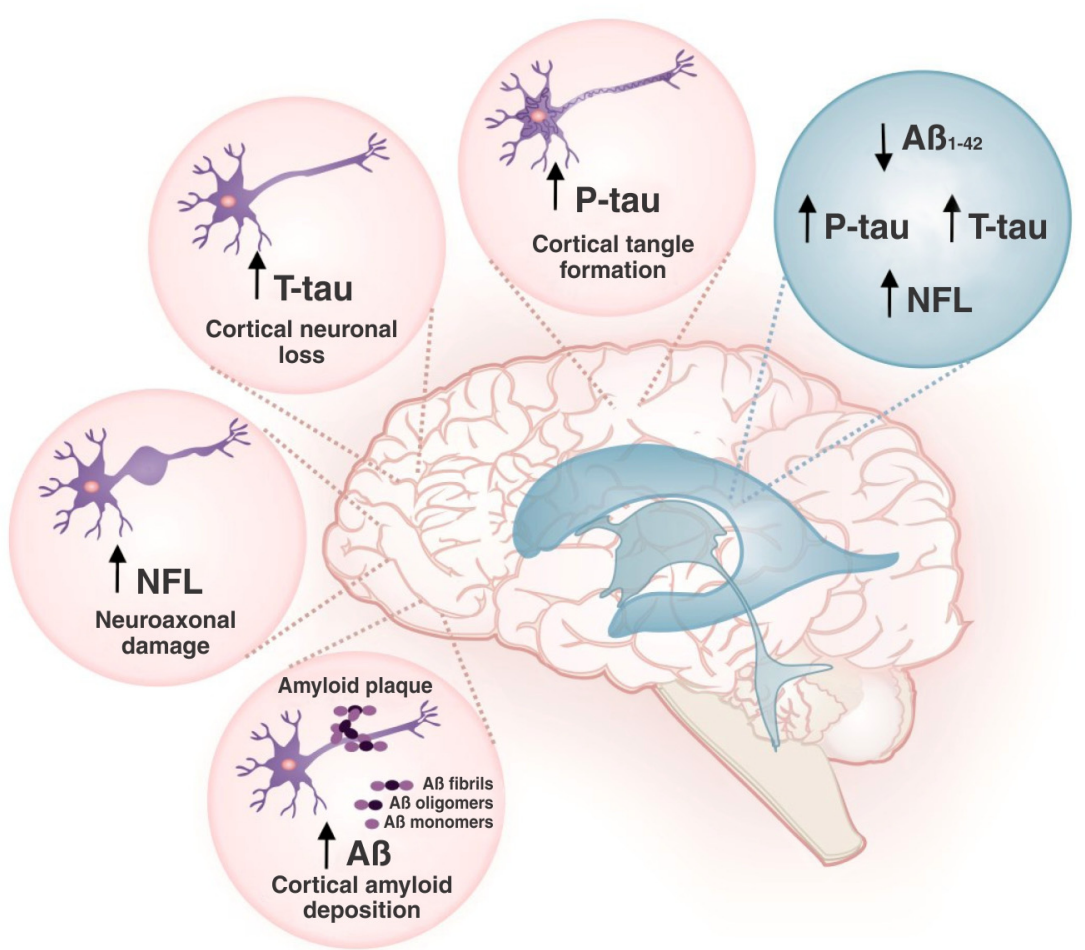

Figure 1 Cerebrospinal fluid (CSF) biosignature for the diagnosis of Alzheimer's disease.In Alzheimer's disease, levels of the 42 -amino acid form of amyloid-beta $\left(A \beta_{42}\right)$ are low, while those of total tau protein (T-tau), phosphorylated tau protein $(P$-tau), and neurofilament light chain (NFL) protein in CSF are high.

positive association of NFL with cognitive impairment. As expected, the $A D$ group presented low levels of $A \beta_{42}$ and high levels of P-tau, T-tau, and NFL compared to the control group. As this study included patients with neurodegenerative disorders, the outcome also emphasizes that CSF NFL levels appear to reflect the intensity of neurodegeneration, and that NFL has the potential to assist in differentiation of AD from FTD. ${ }^{5}$

To date, the biosignature of $A D$ has comprised CSF levels of T-tau, P-tau, and $A \beta_{42}$, as well as plasma T-tau. However, a growing body of evidence suggests NFL is a strong CSF biomarker that should be included in this biosignature.

\section{Acknowledgements}

The authors thank the University of Texas Health Science Center at Houston (UTHealth), Universidade do Extremo Sul Catarinense (UNESC), Conselho Nacional de Desenvolvimento Científico e Tecnológico (CNPq), and Fundação de Amparo à Pesquisa e Inovação de Santa Catarina (FAPESC). TB has received grants from Alzheimer's Association ${ }^{\circledR}$ (AARGDNTF-19-619645).

\section{Disclosure}

The authors report no conflicts of interest.

\section{References}

1 World Health Organization (WHO). New guide for carers of people with dementia [Internet]. 2019 [cited 2019 Aug 26]. http://who.int/ mental_health/neurology/dementia

2 Olsson B, Lautner R, Andreasson U, Öhrfelt A, Portelius E, Bjerke M, et al. CSF and blood biomarkers for the diagnosis of Alzheimer's disease: a systematic review and meta-analysis. Lancet Neurol. 2016;15:673-84.

3 Radanovic M, Oshiro CA, Freitas TQ, Talib LL, Forlenza OV. Correlation between CSF biomarkers of Alzheimer's disease and global cognition in a psychogeriatric clinic cohort. Braz J Psychiatry. 2019;41:479-84.

4 Chapman KR, Bing-Canar H, Alosco ML, Steinberg EG, Martin B, Chaisson $\mathrm{C}$, et al. Mini mental state examination and logical memory scores for entry into Alzheimer's disease trials. Alzheimers Res Ther. 2016;8:9.

5 Olsson B, Portelius E, Cullen NC, Sandelius A, Zetterberg H, Andreasson $U$, et al. Association of cerebrospinal fluid neurofilament light protein levels with cognition in patients with dementia, motor neuron disease, and movement disorders. JAMA Neurol. 2019;76:318-25. 